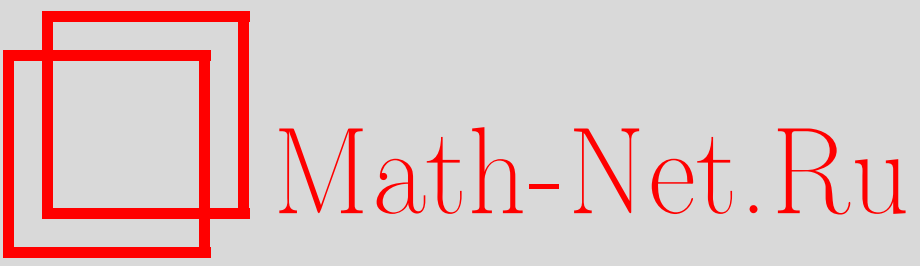

А. М. Каменецкий, Рациональность производящих функций ладейных полиномов и перманентов кронекеровых произведений т'еплицевых матриц и циркулянтов с матрицей $J_{k}, У M H, 2005$, том 60 , выпуск $3,177-178$

DOI: https://doi.org/10.4213/rm1435

Использование Общероссийского математического портала Math-Net.Ru подразумевает, что вы прочитали и согласны с пользовательским соглашением http://www.mathnet.ru/rus/agreement

Параметры загрузки:

IP : 54.164 .48 .24

26 апреля 2023 г., 15:01:30 


\title{
РАЦИОНАЛЬНОСТЬ ПРОИЗВОДЯЩИХ ФУНКЦИЙ ЛАДЕЙНЫХ ПОЛИНОМОВ И ПЕРМАНЕНТОВ КРОНЕКЕРОВЫХ ПРОИЗВЕДЕНИЙ ТЁПЛИЦЕВЫХ МАТРИЦ И ЦИРКУЛЯНТОВ С МАТРИЦЕЙ $J_{k}$
}

\author{
А. М. КАМЕНЕЦКИЙ
}

Известно, что вся классическая теория перечисления перестановок с ограниченными позициями, восходящая еще к Эйлеру, Монмору (1713), Тэйту, Кэли, Мюиру (1878), Люка (1891), Тушару (1934) и Капланскому (1943), сводится к вычислению ладейных полиномов и перманентов $(0,1)$ тёплицевых матриц и $(0,1)$-циркулянтов. Настоящая статья содержит обобщение результатов, полученных автором в [1]-[3].

Пусть $\operatorname{Inj}(S, X)$ - множество вложений множества $S$ в множество $X$.

Пусть $A=\left(a_{i j}\right)_{1 \leqslant i \leqslant m, 1 \leqslant j \leqslant n},-$ прямоуголная матрица надкоммутативным кольцом с единицей, $m \leqslant n, R(x ; z ; A)=1+\sum_{k=1}^{m} x^{k} \sum_{S \subseteq N_{m},|S|=k} \sum_{\sigma \in \operatorname{Inj}\left(S, N_{n}\right)} z^{|\sigma|} \prod_{i \in S} a_{i, \sigma(i)}$ - цикловой ладейньй полином матрицы $A, \operatorname{per}(z ; A)=\sum_{\sigma \in \operatorname{Inj}\left(N_{m}, N_{n}\right)} z^{|\sigma|} \prod_{i \in N_{m}} a_{i, \sigma(i)}$ - цикловой перманент матрицы $A, R(x ; A)=R(x ; 1 ; A), \operatorname{per}(A)=\operatorname{per}(1 ; A)$, где $|\sigma|$ - число циклов инъективного отображения $\sigma, N_{m}=\{1, \ldots, m\}$. Пусть $T_{n}^{(k)}=\left(b_{i j}\right)_{1 \leqslant i, j \leqslant n}, P_{n}=$ $\left(c_{i j}\right)_{1 \leqslant i, j \leqslant n}$

$$
b_{i, j}=\left\{\begin{array}{l}
1, \text { если } j-i=k, \\
0, \text { если } j-i \neq k,
\end{array} \quad c_{i, j}=\left\{\begin{array}{l}
1, \text { если } j-i \equiv 1(\bmod n), \\
0, \text { если } j-i \not \equiv 1(\bmod n) .
\end{array}\right.\right.
$$

Пусть $m_{\bar{\alpha}}(i)$ - число компонент вектора $\bar{\alpha}$, равных $i, i^{\langle l\rangle}=i, \ldots, i(l$ раз $)$,

$$
\begin{aligned}
& G_{t}^{[k]}=\left\{\left(1^{\left\langle l_{1}\right\rangle}, \ldots, t^{\left\langle l_{t}\right\rangle}\right) \mid 0 \leqslant l_{i} \leqslant k, 1 \leqslant i \leqslant t\right\}, \\
& G_{r, t}^{[k]}=\left\{\left(1^{\left\langle l_{1}\right\rangle}, \ldots, t^{\left\langle l_{t}\right\rangle}\right) \in G_{t}^{[k]} \mid \sum_{i=1}^{t} l_{i}=r\right\},
\end{aligned}
$$

$0 \leqslant r \leqslant k t$. Тогда

$$
\begin{aligned}
\left|G_{r, t}^{[k]}\right| & =\underset{x^{r}}{\operatorname{Coef}}\left(1+x+\cdots+x^{k}\right)^{t} \\
& =\sum_{\substack{\lambda_{0}+\lambda_{1}+\cdots+\lambda_{k}=t \\
\lambda_{1}+2 \lambda_{2}+\cdots+k \lambda_{k}=r}} \frac{t !}{\lambda_{0} ! \lambda_{1} ! \cdots \lambda_{k} !}=\sum_{l=0}^{[r /(k+1)]}(-1)^{l}\left(\begin{array}{l}
t \\
l
\end{array}\right)\left(\begin{array}{c}
t+r-l(k+1)-1 \\
t-1
\end{array}\right), \\
\left|G_{t}^{[k]}\right| & =(k+1)^{t} .
\end{aligned}
$$

На множестве $G_{r, t}^{[k]}$ определим квадратную матрицу $\Pi_{r, t}^{[k]}\left(a_{0}, a_{1}, \ldots, a_{t}\right)=\left(a_{\bar{\alpha}, \bar{\beta}}\right)_{\bar{\alpha}, \bar{\beta} \in G_{r, t}^{[k]}}$ следующим образом: $\Pi_{0,0}^{[k]}\left(a_{0}\right)=k ! a_{0}^{k} ;$ если $t \geqslant 1, \bar{\alpha}=\left(1^{\left\langle l_{1}\right\rangle}, \ldots, t^{\left\langle l_{t}\right\rangle}\right) \in G_{r, t}^{[k]}$, то

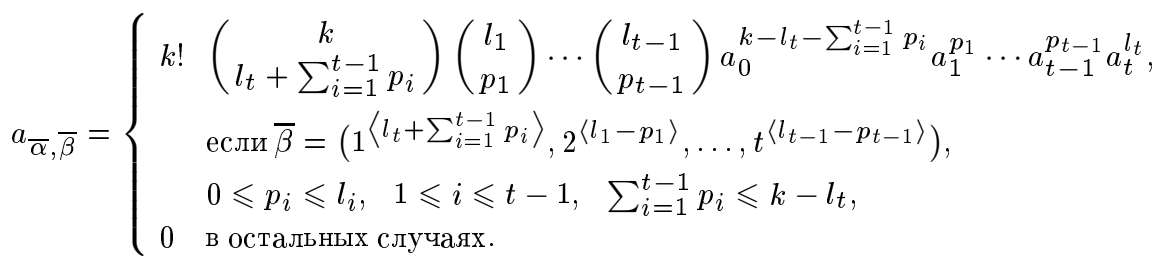


На множестве $G_{t}^{[k]}$ определим квадратную матрицу $K_{t}^{[k]}\left(a_{0}, a_{1}, \ldots, a_{t}\right)=\left(a_{\bar{\alpha}, \bar{\beta}}\right)_{a_{\bar{\alpha}, \bar{\beta}} \in G_{t}^{[k]}}$ следующим образом: $K_{0}^{[k]}\left(a_{0}\right)=\sum_{d=0}^{k} d !\left(\begin{array}{c}k \\ d\end{array}\right)^{2} a_{0}^{d}$; если $t \geqslant 1, \bar{\alpha}=\left(1^{\left\langle l_{1}\right\rangle}, \ldots, t^{\left\langle l_{t}\right\rangle}\right) \in G_{t}^{[k]}$, то

$$
a_{\bar{\alpha}, \bar{\beta}}=\left\{\begin{aligned}
\left(\begin{array}{c}
l_{1} \\
p_{1}
\end{array}\right) & \ldots\left(\begin{array}{c}
l_{t-1} \\
p_{t-1}
\end{array}\right)\left(\begin{array}{c}
k \\
v-\sum_{i=1}^{t-1} p_{i}
\end{array}\right) a_{0}^{v-\sum_{i=1}^{t-1} p_{i}} a_{1}^{p_{1}} \cdots a_{t-1}^{p_{t-1}} \\
& \times \sum_{d=0}^{\min \left(l_{t}, k-v\right)}(d+v) !\left(\begin{array}{c}
k \\
d+v
\end{array}\right)\left(\begin{array}{c}
l_{t} \\
d
\end{array}\right) a_{t}^{d}, \\
& \text { если } \bar{\beta}=\left(1\left\langle k-v+\sum_{i=1}^{t-1} p_{i}\right\rangle, 2^{\left\langle l_{1}-p_{1}\right\rangle}, \ldots, t^{\left\langle l_{t-1}-p_{t-1}\right\rangle}\right), \\
& 0 \leqslant p_{i} \leqslant l_{i}, \quad 1 \leqslant i \leqslant t-1, \quad \sum_{i=1}^{t-1} p_{i} \leqslant v \leqslant k, \\
0 \quad & \text { в остальных случаях. }
\end{aligned}\right.
$$

Если $X$ - конечное множество мощности $n$, то обозначим символом $\mathscr{L}$ (в контексте конкретного рассматриваемого множества $X$ ) некоторую фиксированную биекцию $X$ на $N_{n}$. Пусть $J_{k}$ - квадратная матрица порядка $k$, все элементы которой равны 1.

Теорема 1. Пусть $0 \leqslant r \leqslant t, a_{-r}, a_{-r+1}, \ldots, a_{-r+t}-$ элементы из коммутативного кольца с единицей. Тогда для всех $n \geqslant 1$

$$
\begin{aligned}
& R\left(x ;\left(\sum_{i=0}^{t} a_{-r+i} T_{n}^{(-r+i)}\right) \otimes J_{k}\right) \\
& \quad=\sum_{\substack{\gamma \in \bigcup_{l=r k}^{k t} G_{l, t}^{k k]} \\
\{\bar{\gamma}\} \supseteq\left\{1^{\langle k\rangle}, \ldots, r^{k k\rangle}\right\}}}\left(K_{t}^{[k]}\left(a_{-r} x, a_{-r+1} x, \ldots, a_{-r+t} x\right)\right)^{n}\left[\mathscr{L}\left(1^{\langle k\rangle}, \ldots, r^{\langle k\rangle}\right) \mid \mathscr{L}(\bar{\gamma})\right], \\
& \operatorname{per}\left(\left(\sum_{i=0}^{t} a_{-r+i} T_{n}^{(-r+i)}\right) \otimes J_{k}\right) \\
& \quad=\left(\left(\Pi_{r k, t}^{[k]}\left(a_{-r}, a_{-r+1}, \ldots, a_{-r+t}\right)\right)^{n}\left[\mathscr{L}\left(1^{\langle k\rangle}, \ldots, r^{\langle k\rangle}\right) \mid \mathscr{L}\left(1^{\langle k\rangle}, \ldots, r^{\langle k\rangle}\right)\right],\right. \\
& R\left(x ;\left(\sum_{i=0}^{t} a_{-r+i} P_{n}^{-r+i}\right) \otimes J_{k}\right)=\operatorname{Tr}\left(\left(K_{t}^{[k]}\left(a_{-r} x, a_{-r+1} x, \ldots, a_{-r+t} x\right)\right)^{n}\right), \\
& \operatorname{per}\left(\left(\sum_{i=0}^{t} a_{-r+i} x^{i} P_{n}^{-r+i}\right) \otimes J_{k}\right)=\sum_{l=0}^{k t}\left(\operatorname{Tr}\left(\left(\Pi_{l, t}^{[k]}\left(a_{-r}, a_{-r+1}, \ldots, a_{-r+t}\right)\right)^{n}\right)\right) x^{l n} .
\end{aligned}
$$

Теорема 2. Пусть $k \geqslant 1, t \geqslant 1, a_{0}, a_{1}, \ldots, a_{t}-$ переменные, $1 \leqslant n \leqslant t$. Тогда

$$
\begin{aligned}
& \operatorname{Tr}\left(\left(\frac{\partial}{\partial a_{0}} K_{t}^{[k]}\left(a_{0}, a_{1}, \ldots, a_{t}\right)\right) \cdot\left(K_{t}^{[k]}\left(a_{0}, a_{1}, \ldots, a_{t}\right)\right)^{n-1}\right) \\
& \quad=\operatorname{Tr}\left(\left(\frac{\partial}{\partial a_{n}} K_{t}^{[k]}\left(a_{0}, a_{1}, \ldots, a_{t}\right)\right) \cdot\left(K_{t}^{[k]}\left(a_{0}, a_{1}, \ldots, a_{t}\right)\right)^{n-1}\right) .
\end{aligned}
$$

\section{СПИСОК ЛИТЕРАТУРЫ}

[1] А. М. Каменецкий // Деп. в ВИнИТИ 14.08.1990, № 4620-В90; РЖМат. 1 А378. 1991. [2] А. М. Каменецкий // Деп. в ВИНИТИ 19.08.1991, № 33487-В91; РЖКМат. 12 А363. 1992. [3] А. М. Каменецкий // Деп. в ВИНИТИ 28.08.1992, № 2695-В92; РЖМат. 2 А314. 1993. 